\title{
Analysis of the magnetic field discontinuity at the potential field source surface and Schatten Current Sheet interface in the Wang-Sheeley-Arge model
}

Article

Published Version

McGregor, S. L., Hughes, W. J., Arge, C. N. and Owens, M. J. (2008) Analysis of the magnetic field discontinuity at the potential field source surface and Schatten Current Sheet interface in the Wang-Sheeley-Arge model. Journal of Geophysical Research, 113 (A8). A08112. ISSN 0148-0227 doi: https://doi.org/10.1029/2007JA012330 Available at https://centaur.reading.ac.uk/5825/

It is advisable to refer to the publisher's version if you intend to cite from the work. See Guidance on citing.

Published version at: http://dx.doi.org/10.1029/2007JA012330

To link to this article DOI: http://dx.doi.org/10.1029/2007JA012330

Publisher: American Geophysical Union

All outputs in CentAUR are protected by Intellectual Property Rights law, including copyright law. Copyright and IPR is retained by the creators or other copyright holders. Terms and conditions for use of this material are defined in the End User Agreement. 


\section{www.reading.ac.uk/centaur}

\section{CentAUR}

Central Archive at the University of Reading

Reading's research outputs online 


\title{
Analysis of the magnetic field discontinuity at the potential field source surface and Schatten Current Sheet interface in the Wang-Sheeley-Arge model
}

\author{
S. L. McGregor, ${ }^{1}$ W. J. Hughes, ${ }^{1}$ C. N. Arge, ${ }^{2}$ and M. J. Owens ${ }^{1}$ \\ Received 7 February 2007; revised 27 October 2007; accepted 6 March 2008; published 22 August 2008.
}

[1] The Wang-Sheeley-Arge solar wind model makes use of coupled potential field source surface (PFSS) and Schatten Current Sheet (SCS) models to reconstruct the coronal magnetic field on the basis of the observed line-of-sight photospheric magnetic field and a 1D kinematic code to propagate the solar wind to $1 \mathrm{AU}$. The source surface serves as the outer boundary of the PFSS model and the inner boundary of the SCS model. Known discontinuities arise in the tangential components of the magnetic field across this surface owing to differences in the imposed boundary conditions (Wang et al., 1998). Here we introduce a more flexible coupling between the two models, which considerably reduces the discontinuous behavior of the magnetic field across the model interface surface, to investigate the effects and importance of these kinks on the accuracy of the model's solar wind speed predictions at 1 AU. A detailed analysis of select Carrington rotations shows that removing the kinks can lead to changes in connectivity, creating different source regions for the solar wind. These changes lead to significantly improved predictions of solar wind structures at 1 AU some of the time, but most of the time, the kinks do not affect the predicted solar wind speed. This improvement is born out statistically by increases in the prediction skill scores of both solar wind velocity $(1.7 \%)$ and interplanetary magnetic field polarity $(1.4 \%)$ at $1 \mathrm{AU}$.

Citation: McGregor, S. L., W. J. Hughes, C. N. Arge, and M. J. Owens (2008), Analysis of the magnetic field discontinuity at the potential field source surface and Schatten Current Sheet interface in the Wang-Sheeley-Arge model, J. Geophys. Res., 113, A08112, doi:10.1029/2007JA012330.

\section{Introduction}

[2] Since the late sixties potential field approximations have been used to infer the magnetic structure of the solar corona [Schatten et al., 1969; Altschuler and Newkirk, 1969]. The models have since evolved into widely accepted tools for understanding heliospheric phenomena [e.g., Wang and Sheeley, 1992; Zhao and Hoeksema, 1993, 1995; Neugebauer et al., 2002; Arge et al., 2004; Schrijver et al., 2005], and have been implemented into operational situations for prediction of solar wind conditions near earth such as the Wang-Sheeley-Arge (WSA) solar wind model, which runs routinely at NOAA Space Weather Prediction Center (www.swpc.noaa.gov/ws/). The Wang-SheeleyArge [Arge and Pizzo, 2000; Arge et al., 2003, 2004] model uses a potential field based approximation to allow a computationally efficient method of estimating the coronal magnetic field configuration. This model has been shown through direct comparisons with coronal observations [Neugebauer

\footnotetext{
${ }^{1}$ Center for Integrated Space Weather Modeling, Boston University, Boston, Massachusetts, USA.

${ }^{2}$ Air Force Research Laboratory/Space Vehicles Directorate, Kirtland Air Force Base, New Mexico, USA.
}

Copyright 2008 by the American Geophysical Union. 0148-0227/08/2007JA012330\$09.00 et al., 1998; de Toma et al., 2005; de Toma and Arge, 2006] to compare favorably with those derived by more computationally intensive MHD models.

[3] Given the wide use of the WSA model, it is important to make it as robust as possible. In this paper we investigate the interface between the potential field source surface (PFSS) and the Schatten Current Sheet (SCS) models, which together provide the coronal reconstruction in the WSA model. Conflicting boundary conditions cause tangential discontinuities in field lines as they traverse the boundary [Wang and Sheeley, 1988]. While Wang and Sheeley [1988] argue that this has minimal effect on the overall coronal solution, a detailed analysis of its actual importance has not been done. Zhao and Hoeksema [1993, 1995] have used least square fits at the boundary to successfully reduce the kinks. In this paper, we introduce a simple more flexible coupling that substantially reduces field line kinks and investigate the affects this new coupling, and therefore field line kinks, have on solar wind velocity predictions at earth. Kinks in the magnetic field lines are most pronounced on field lines close to the current sheet; thus we might expect changes to be most commonly observed during periods when the current sheet remains close to the ecliptic. For this reason we chose to compare these predictions to Wind observations for the solar minimum year of 1995. This is also a period when there are few 

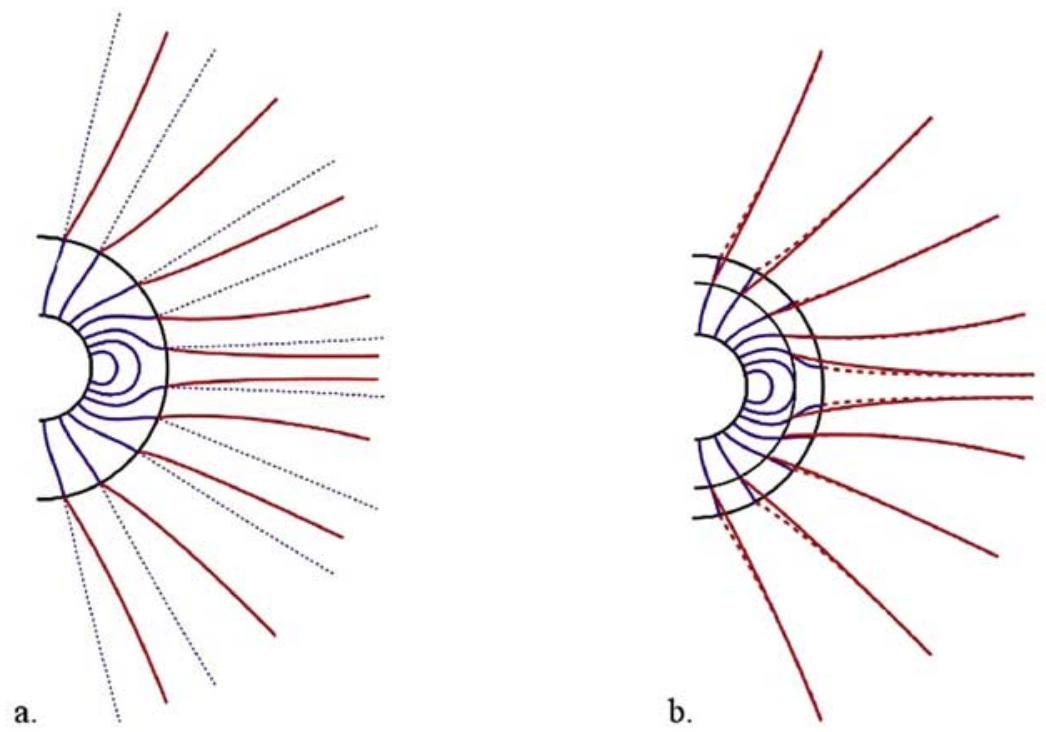

Figure 1. A sketch of the coronal magnetic field solution of the WSA model. Solid blue lines indicate the inner PFSS model. (a) Blue dotted lines outside of the source surfaces show radial field lines, while the red solid lines give the solution from the SCS model. (b) Red dotted lines from the source surface show the original SCS solution. Solid red lines beginning at the interface surface of the SCS model with the new coupling show a noticeable reduction of the discontinuities or "kinks" in the field lines.

solar transient events to complicate the comparisons. Arge et al. [2003] found this time period particularly difficult to accurately predict solar wind parameters. We believe this difficulty is primarily due to the flatness of the current sheet, which causes the sub-earth track to remain close to the steep gradients between the fast and slow wind. Thus even minor latitudinal uncertainties in its position (e.g., because of uncertainties in the strengths of the polar field) can easily result in pronounced over- or under-predictions of solar wind speed. 1995 therefore serves as a good time to study the relative importance of the kinked field lines against other uncertainties such as the strength of the polar field or the current sheet location.

\section{The Magnetic Field Model}

[4] The WSA model is a combination of three models. The first two are the Potential Field Source Surface (PFSS) model and Schatten Current Sheet (SCS) model, which are used in combination to derive, respectively, the inner and outer coronal magnetic field as illustrated in Figure 1a. The third model is a 1D modified kinematic code to propagate the solar wind out from $5 \mathrm{R}_{\odot}$ to $1 \mathrm{AU}$ [Arge and Pizzo, 2000] accounting for stream interactions in an ad hoc fashion. Within the source surface the WSA-derived corona, indicated by the solid blue lines in Figure 1a, is determined by the PFSS model which assumes that the magnetic field can be described as a scalar potential, satisfying Laplace's equation. This implies that the curl of the magnetic field is zero, and hence no currents flow in this region. This scalar potential is uniquely determined given well-defined boundary conditions on the inner and outer boundaries. On the inner boundary (photosphere) the normal derivative of the scalar potential is specified (i.e., the radial component of the magnetic field), calculated from line-of-sight photospheric magnetic field measurements [Wang and Sheeley,
1992]. At the outer boundary (i.e., the source surface) the scalar potential is set to zero, thus forcing the magnetic field to be radial. This additional constraint of a radial magnetic field at the source surface is imposed to mimic the behavior of the magnetic field in the outer corona, which, being frozen into the coronal plasma, is dragged out radially by the solar wind [Altschuler and Newkirk, 1969; Schatten et al., 1969].

[5] The SCS model derives the magnetic field topology of the upper corona. The radial component of the magnetic field at the source surface provides the inner boundary condition, leaving the tangential magnetic field components unspecified. The SCS model assumes no volumetric currents in the outer corona except where the radial component of the magnetic field reverses. The assumption is valid as the plasma $\beta$ is low in the outer corona, meaning any currents result in large $\mathrm{J} \times \mathrm{B}$ forces which, in the absence of significant thermal pressure, quickly reorganize the field. Thus currents can only flow where $\mathrm{JxB}$, and hence the magnetic field strength, is near zero (i.e., in regions where the field polarity changes). The SCS model accomplishes this mathematically by first (temporarily) reorienting the field at the source surface to give the entire solar field the same polarity. A potential solution, with the field vanishing at infinity, is then solved in the outer corona, effectively solving for a magnetic monopole. This solution is then truncated at $5 \mathrm{R}_{\odot}$ and the correct magnetic field polarity is restored to the field lines, thus retaining the lack of volumetric currents save at the polarity inversion of the field [Schatten, 1971].

[6] In summary, the PFSS model constrains the field to be radial at the source surface, whereas the SCS model does not require the components of the field tangential to its inner boundary (i.e., at the source surface) to be zero, only requiring the radial field components to specify a unique solution. In general, this discontinuity in the latitudinal and 

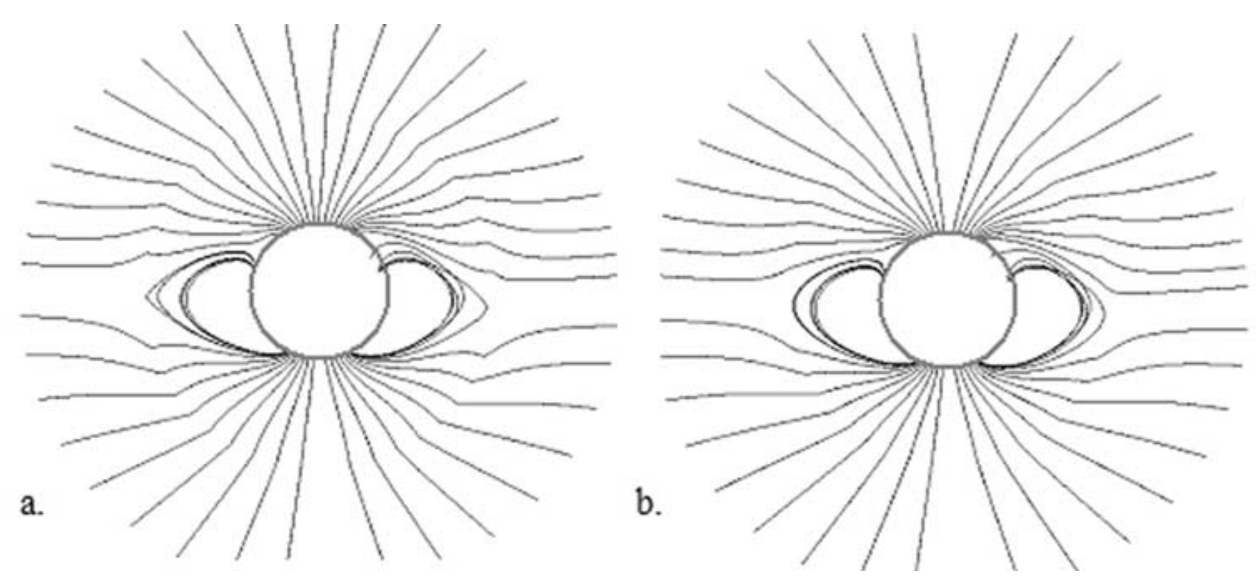

Figure 2. Magnetic fields calculated by the original WSA model (a) and the modified WSA model (b) for CR 1902. Kinks in the magnetic field lines have not been completely eliminated in the Modified WSA model but are greatly reduced when compared with the original WSA model.

longitudinal components means that the magnetic field lines will be "kinked" at the source surface, implying that a surface current flows on the source surface. These "kinks" are evident in Figure 1a at the intersection between the solid red and solid blue lines.

[7] In order to minimize the currents flowing on the interface surface, and hence derive a more realistic model of the solar coronal magnetic field by reducing the unphysical magnetic field kinks, we propose a more flexible coupling of the PFSS and SCS models. We introduce a new "interface surface" between the two models, inside the outer boundary of the PFSS model (see Figure 1b). In this approach, the PFSS model is solved as before with a radial field at its outer surface. However, instead of using the radial field components at the outer boundary of the PFSS model as the input into the SCS model, we use the radial field components at the interface surface, which lies below the PFSS model's outer boundary. Here the field lines have a more 'natural' configuration (i.e., not forced to be radial). The tangential components of the magnetic field are, in general, nonzero on both sides of the interface surface, but they do not necessarily match. However, as will be seen, this methodology tends to significantly reduce the kinks in the modeled field lines.

[8] For this study we use $2.5^{\circ}$ resolution synoptic maps of the photospheric line-of-sight magnetic field from Mount Wilson Solar Observatory (MWO), interpolated from $4^{\circ}$, as input into the model. The polar magnetic fields were corrected by adjusting the polar fields to fit a trend-line, which was derived from past observations of the poles during times of high solar $b$ angle [Arge and Pizzo, 2000; Arge et al., 2004]. We use spherical harmonics to solve Laplace's equation, using 72 harmonics for the $2.5^{\circ}$ synoptic maps. We use the approach taken by Wang and Sheeley [1988, 1992, and 1995] to solve the spherical harmonic expansion of the PFSS and SCS models.

\section{Determining Optimal Model Parameters}

[9] Altschuler and Newkirk [1969] concluded that $2.5 \mathrm{R}_{\odot}$ was an optimal source surface, consistent with Hoeksema [1984]. Since then, this value has been commonly used for the source surface [e.g., Arge et al., 2004; Neugebauer et al., 2002]. However, by introducing the interface surface, we now have to specify optimal values for two radii. As the height of the boundary radius $\left(\mathrm{R}_{\mathrm{b}}\right)$ increases more of the solar magnetic flux closes inside this surface leaving less total open solar magnetic flux. On the other hand, as the interface radius $\left(\mathrm{R}_{\mathrm{i}}\right)$ moves sunward more solar magnetic flux crosses this surface and enters the SCS model and hence is open to the heliosphere. In order to maintain the amount of open solar magnetic flux as previously calculated by the original WSA model [Wang and Sheeley, 1988], we find pairs of radii which best preserve the absolute open magnetic flux, thus helping to minimize the changes between the original and modified models to make the best comparison. Radii are also chosen which decrease the current flowing at the model interface. Thus there are two constraints to finding the best pair of radii for the outer boundary of the PFSS model and that of the interface surface, the reduction of the total current flowing on the interface surface, and the continuance of the original quantity of open solar magnetic flux.

[10] To determine the optimum combination of $R_{i}$ and $R_{b}$, we used 28 Carrington rotations from different parts of the solar cycle, ranging from solar minimum to solar maximum between Carrington rotations $1890-1988$. We varied $R_{i}$ between $2.0 \mathrm{R}_{\odot}$ and $2.6 \mathrm{R}_{\odot}$ in steps of. $1 \mathrm{R}_{\odot}$. For each value of $R_{i}$ we varied $R_{b}$ from $R_{i}$ to $3.0 R_{\odot}$ in steps of. $1 R_{\odot}$, resulting in 57 different combinations of $R_{i}$ and $R_{b}$. For each rotation a matrix was constructed and values of the sum of the current density over the interface, as well as the absolute open magnetic flux, are recorded for each pair of radii. A few pairs of radii which best fit our criteria are chosen for each Carrington Rotation. The most frequently occurring pair was $\mathrm{R}_{\mathrm{b}}=2.6 \mathrm{R}_{\odot}$ and $\mathrm{R}_{\mathrm{i}}=2.3 \mathrm{R}_{\odot}$. This combination preserves the absolute magnetic flux for all rotations to within $7 \%$, with an average of $6 \%$, and reduces the currents flowing along the surface by a factor of 5 . Figure 2 shows the WSA magnetic field lines for Carrington rotation 1902 projected onto a meridional cut plane. The two panels show magnetic field lines from before (Figure 2a) and after (Figure 2b) the introduction of the interface surface. The black circle has a radius of $1 R_{\odot}$. 
While the kinks in the field lines have not been completely eliminated, they have been greatly reduced.

\section{Solar Wind Velocity Predictions}

[11] The WSA model uses two parameters to predict solar wind velocities at $5 \mathrm{R}_{\odot}$, the flux tube expansion factor, given by the amount that the magnetic flux tube expands in solid angle between the photosphere and the interface surface [Wang and Sheeley, 1990], and the distance of the magnetic field line foot point to the nearest coronal hole boundary. Through systematic testing of various relationships between expansion factors, coronal hole boundary distance, and solar wind velocity, Arge et al. [2003] calculated the velocity equation used on the $5 \mathrm{R}_{\odot}$ surface. However, Arge et al. [2003] calculated the velocity equation using $5^{\circ}$ Kitt Peak National Solar Observatory (NSO) data as input into the model. Since in this study we use $2.5^{\circ}$ Mount Wilson Solar Observatory (MWO) synoptic maps with corrected poles for both the original and modified WSA, we recalculate the numerical coefficients in the original expression by Arge et al. [2003] We recalculate the equation to obtain the best fit to Wind spacecraft observations of solar wind velocity for the Carrington rotations 1890-1904 (approximately covering the year 1995) using the original WSA model. To best compare the differences introduced solely by the reduction of the kinks, this velocity equation was then used for both the original and modified WSA models. The resulting expression is

$V=265.0+\left(1.5 /\left(1.0+f_{s}\right)^{1 / 2.5}\right) *\left(5.8-4.0 * \exp \left(-\left(\theta_{b} / 2.5\right)^{2.0}\right)\right)^{3.5}$

where $\mathrm{f}_{\mathrm{s}}$ is the expansion factor as defined by Wang and Sheeley [1990], and $\theta_{\mathrm{b}}$ is the angular distance from the magnetic field foot point to the coronal hole boundary. This relationship is used to calculate velocities at $5 \mathrm{R}_{\odot}$. The velocities are then propagated using the same kinematic code out to 1 AU [Arge and Pizzo, 2000].

\section{Results and Model Evaluation}

[12] Figure 3 compares the original and modified WSA model predictions with the solar wind speed observed by the Wind spacecraft at earth for five Carrington rotations. These five rotations were chosen to highlight differences between the predictions of the two models as compared with solar wind observations. Recall that this interval was one of the most difficult to model correctly. In each panel the Wind observations are shown as a black line while the original and modified WSA model results are shown as blue triangles and red squares respectively. As per Arge et al. [2003], the velocities are propagated kinematically from equispaced origins on the 21.5 solar radii sphere with the limitation that the fast wind may not overtake the slow wind. Thus the model speed predictions are not equispaced in time at $1 \mathrm{AU}$; the longer intervals between predictions occur when the predicted speed decelerates.

[13] Figure 3a shows CR 1902. The solar wind velocity remained below $400 \mathrm{~km} / \mathrm{s}$ for much of this rotation, with the exception of the period between November 1 and 9 when two distinct high-speed streams were observed, with veloc- ity peaking on November 1 and 6. The original WSA model predicts high-speed solar wind continuously from October 31 until November 5. Thus, while it predicts the occurrence of high-speed flow, it only predicts a single extended stream that ends earlier than observed. The modified WSA predicts two distinct streams with the speed minimum between the two streams occurring early on November 5 , close to that observed by Wind. However, the predicted second stream is still shorter lived than is observed. For the remainder of the rotation the predictions of the two models do not differ significantly; both predict slightly higher than observed speeds during the second half of the rotation.

[14] The previous rotation, CR 1901, is shown in Figure 3b. This rotation has a more variable solar wind speed. The double high-speed stream observed in early November in Carrington rotation 1902, appears as a single interval of fast flow that extended from October 4 to 9 in Carrington rotation 1901. This high-speed stream is predicted very well by the modified WSA, which correctly predicts both the onset time and maximum speed. This model predicts the continuation of the high-speed stream, but predicts that the speed decreases about a day earlier than observed by Wind. The original WSA model does not capture the first stream nearly as well, with only a single point above $500 \mathrm{~km} / \mathrm{s}$ in the first half of October. During the second half of this rotation both models predict the speed enhancement observed on October 20, but with an onset about 12 hours later than observed by Wind.

[15] Figure 3c shows Carrington rotation 1896. The solar wind velocity for this time period is clearly described by two consecutive high-speed streams that are flanked by a lower speed at the beginning and end of the rotation. The observations show that the first high-speed stream peaks on May 24 after sharply rising in speed from around $350 \mathrm{~km} / \mathrm{s}$ to over $700 \mathrm{~km} / \mathrm{s}$ in less than a day. Both models incorrectly predict the onset of this stream almost a day early, and neither predicts speeds higher than $600 \mathrm{~km} / \mathrm{s}$ for this stream. After the peak, the original WSA model more closely predicts the decline of the solar wind speed from May 26 to 29. The modified WSA model predicts speeds about $50 \mathrm{~km} / \mathrm{s}$ slower than observed for this same region. The onset of the second high-speed stream occurs abruptly on May 30, and lasts for 5 days before it begins to decline on June 4. Both models accurately predict an abrupt onset to this stream, but while the modified WSA model predicts the onset time accurately, the original WSA model predicts it about a day early. For the remainder of the rotation both models predict similar velocities for the high-speed stream and the slower speed winds at the end of the rotation. Both predict the decline of the solar wind speed to begin on June 2, almost 36 hours earlier than observed.

[16] Carrington rotation 1893 is shown in Figure 3d. Two high-speed streams are seen in the observations. The first high-speed stream peaks from March 1 to 3, with a speed of about $650 \mathrm{~km} / \mathrm{s}$. The second high-speed stream averages about $700 \mathrm{~km} / \mathrm{s}$ starting on March 11 until 14 . Both models predict relatively slow solar wind velocities for the first third of the rotation, completely missing the first observed high-speed stream. Around March 5, the velocities predicted by both the original and the modified WSA models quickly increase to above $400 \mathrm{~km} / \mathrm{s}$, to match the speeds seen by the Wind spacecraft. The 
a.

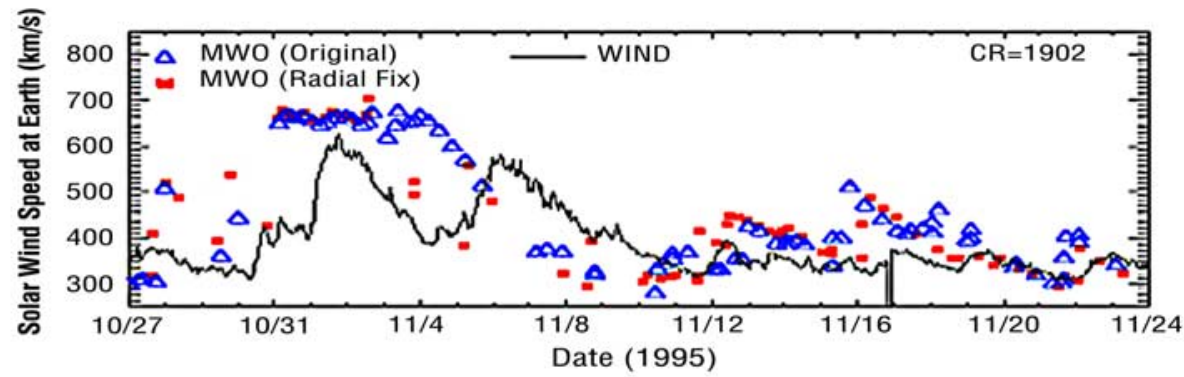

b.

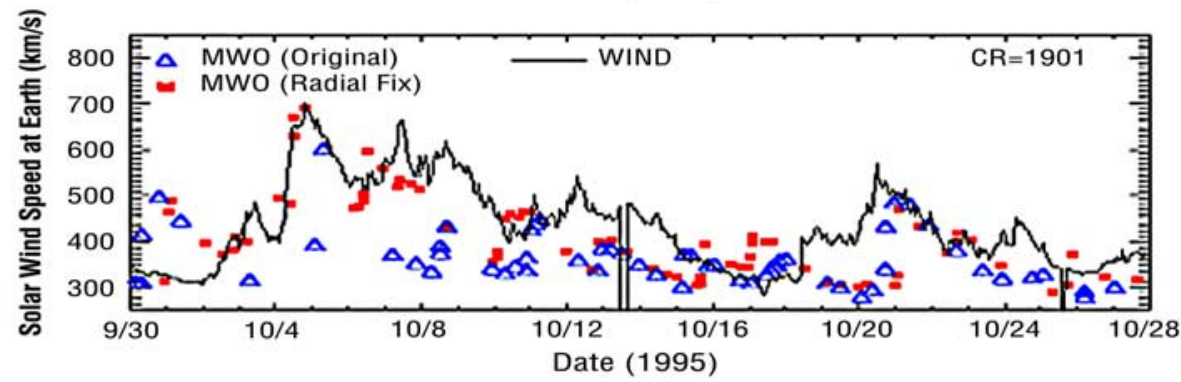

c.
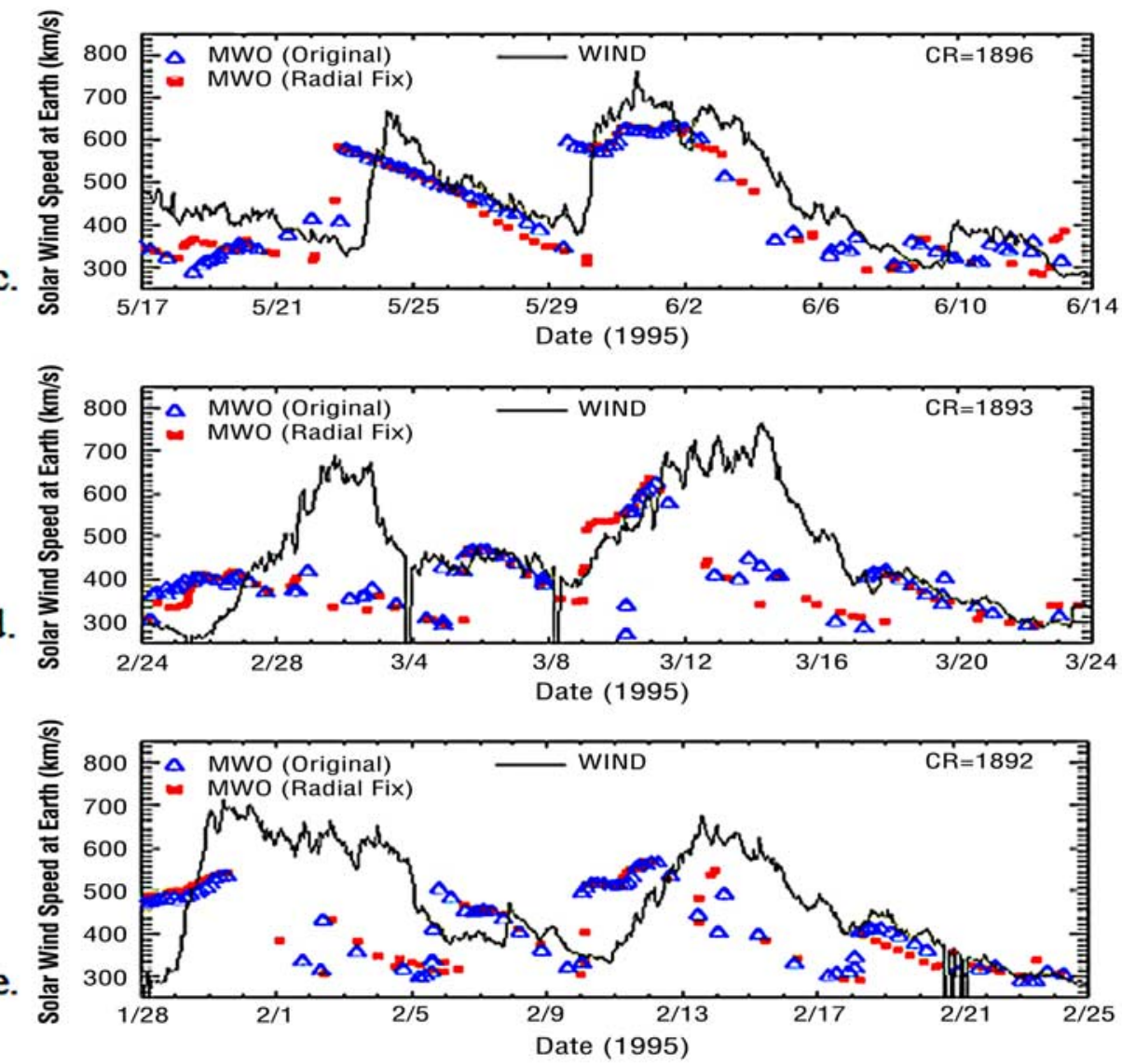

Figure 3. Velocity predictions from the original WSA (blue) and modified WSA (red) for five CRs in 1995 compared to wind observations. Time goes from left to right along the $x$ axis, while velocities range from 250 to $800 \mathrm{~km} / \mathrm{s}$ along the $y$ axis.

original WSA model continues to predict low speeds, slowing to around $300 \mathrm{~km} / \mathrm{s}$ on March 10, when the velocity predictions increase quickly to peak just over $600 \mathrm{~km} / \mathrm{s}$ on March 11. The modified WSA model better predicts the rise in solar wind velocity seen by Wind between March 8 and 11, peaking at the same velocity and time that the original WSA model does. Both models give the same solar wind predictions for the rest of the rotation, declining from March 11 to 17, and thus missing the rest of the second high-speed stream. The predictions abruptly jump in magnitude again on March 17 to match the observations for the rest of the rotation.

[17] Carrington rotation 1893 is a good example of how changes in magnetic connectivity can affect solar wind 

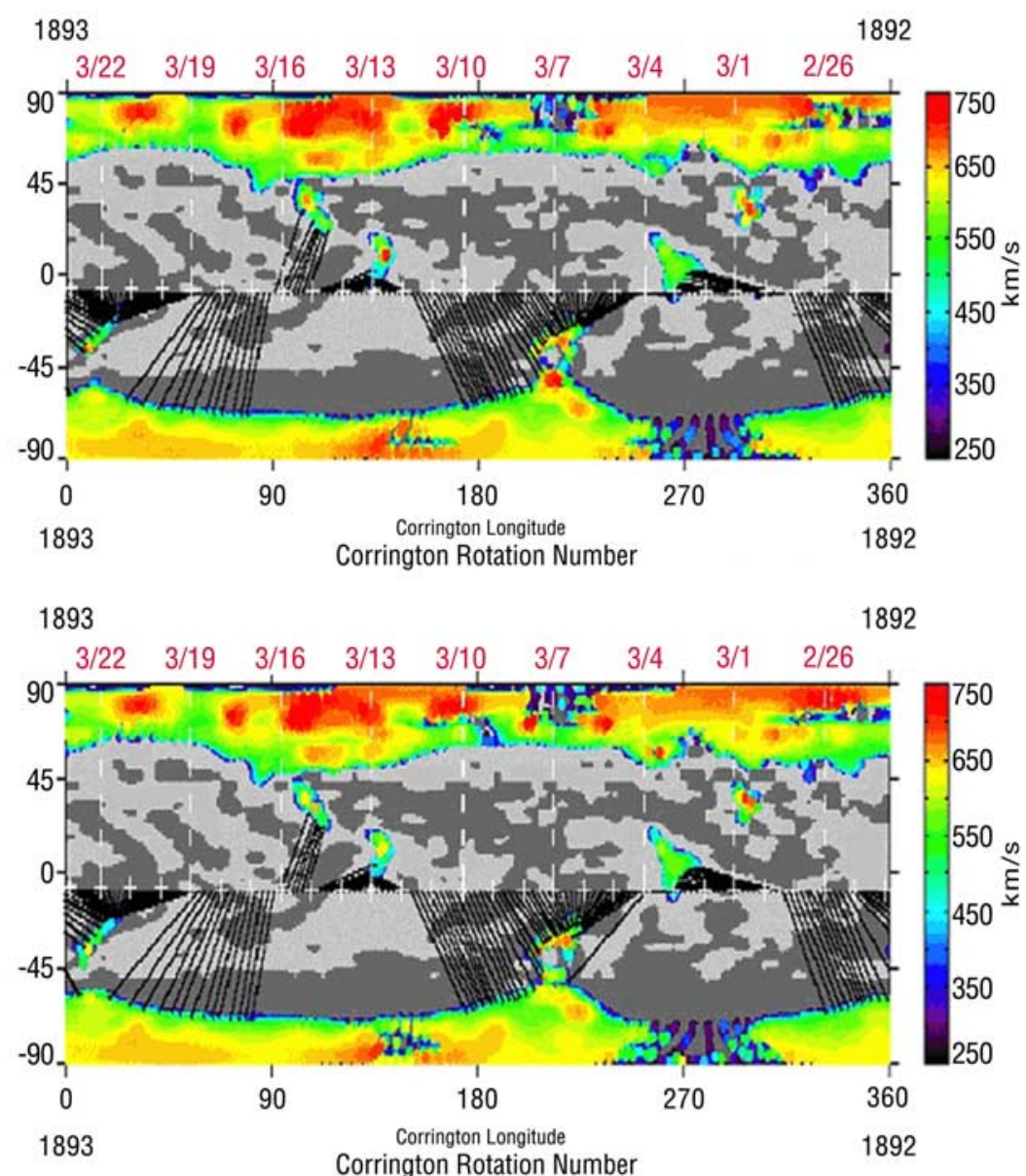

Figure 4. Maps of the photospheric polarity and the location of the foot points of open magnetic field for the original WSA (top) and modified WSA (bottom) for CR 1893. Foot points are colored with solar wind speed. White crosses indicate the sub-earth location, while the black lines show how each sub-earth field line connects at the photosphere.

velocity predictions. The two panels in Figure 4 show maps of the photosphere for the original (upper) and modified (lower) WSA models for Carrington rotation 1893. Regions where the magnetic field lines extend beyond the source surface (i.e., open flux regions) are colored with the solar wind velocity predicted on those field lines at $5 \mathrm{R}_{\odot}$. Regions where the field lines close within the source surface are light or dark gray depending on whether the magnetic polarity is positive or negative respectively. The white crosses are the sub-earth points and the black lines indicate where a field line from that sub-earth point (on a 5 solar radii sphere) connects to the solar surface (i.e., the source region of solar wind predicted at earth). The dates indicate when that particular Carrington longitude was located at central meridian, note that time runs from right to left. The top panel shows the results for the original WSA model (blue triangles on Figure 3d), and the lower panel is for the modified WSA (the red squares on Figure $3 \mathrm{~d}$ ).

[18] Close inspection of Figure 4 reveals subtle but significant changes in the connectivity of the sub-earth points between the original and modified models. On March 4 through 5 the field lines show an important change in connectivity between the original and modified models. The sub-earth points are magnetically connected to different parts of the southern hemisphere coronal hole extension. Figure 5 shows a close-up of this region. In Figure 5a the field lines connected to the sub-earth point around March 4 magnetically connect to the solar surface only at the top rounded part of the coronal hole extension seen at a Carrington longitude of about $210^{\circ}$. In Figure $5 \mathrm{~b}$ these same field lines connect to the solar surface at lower solar latitudes, connecting along the right edge of that coronal hole extension, and mapping deeper into it. The most significant difference is seen in the field line connecting the sub-earth point on March 4. In Figure 5a this point connects to the bottom of the low latitude hole found in the northern hemisphere at a Carrington Longitude of about $270^{\circ}$. The black connecting line is almost horizontal and barely visible. In sharp contrast, in Figure 5b this point connects to the base of the coronal hole extension at a southern latitude of about $55^{\circ}$. A closer look at the coronal hole extension itself reveals a slightly different configuration of the open field foot points. This reconfiguration of the field lines connected to the sub-earth points on the March 4 and 5, mapping to a slightly different coronal hole extension, resulted in improvements to the velocity profile seen between March 8 and 10 (Figure 3d). This analysis demonstrates that changes in magnetic connectivity can have 


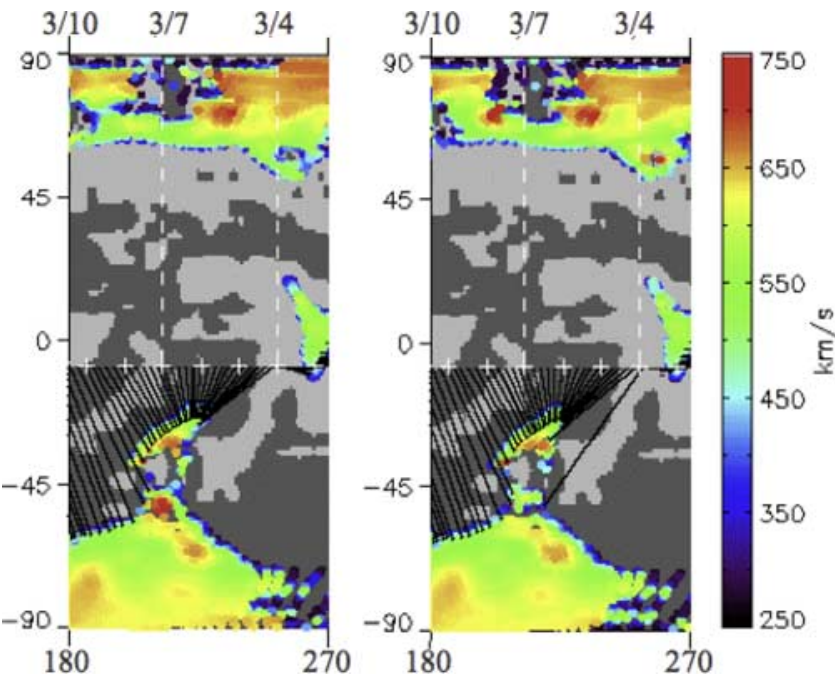

Figure 5. Zoomed in view of Figure 4 focusing around March 3 to 7 for the original WSA (a) and modified WSA (b). Colored dots indicated the velocity of the solar wind at 5 solar radii. White crosses are the sub-earth points. Black lines show the magnetic connectivity of the sub-earth points to the photosphere.

very significant changes in the predicted solar wind speed. Reconfigurations can also be found that do not significantly alter the predicted solar wind speed, but do result in a different source region for the solar wind.

[19] Figure 6 provides a different perspective for observing the predicted velocity differences (for March 8 and 10) or lack there of (for March 20 through 22). This figure shows the solar wind velocity predictions at $1 \mathrm{AU}$ for the original (top) and modified (bottom) WSA models for Carrington rotation 1893 (Figure 3d) as a function of the latitude above and below the ecliptic (vertical axis) and predicted arrival date of the solar wind at 1AU (horizontal axis) with time running from left to right as in Figure 3d.

[20] Velocities are given by a color scale that varies from $200 \mathrm{~km} / \mathrm{s}$ (dark blue) to $800 \mathrm{~km} / \mathrm{s}$ (white) and are calculated using the same 1D kinematic method described earlier. The velocity observed by the Wind spacecraft is shown, using the same color scale, by the strip at the top of the image.

[21] Overall the features seen in both panels are very similar. Both models show a similar broad, $10-20^{\circ}$ latitude wide, undulating slow speed flow near the ecliptic. In general, higher speeds are predicted at high latitudes, with the exception of a region around March 7 in the southern hemisphere, and a region around March 14 in the northern hemisphere. There are also two regions of higher-speed flows near the ecliptic within the slow undulating flow, one around March 6 and another around March 10.

[22] For the Original WSA, the high-speed stream within the slow flow occurs from March 9 to 13. In the modified WSA this higher-speed stream occurs from March 8 to 13 . This difference corresponds to the improvements previously indicated in Figure 3d on March 8 and 9 by the Modified WSA model. The models are correctly calculating the presence of a high-speed stream here, but are transitioning back into the slow solar wind around the current sheet too quickly. This high-speed stream within the slow speed has a source region in the coronal hole extension in the southern hemisphere near Carrington longitude 210 in Figure 4. The coronal hole extension, with an inwardly oriented radial magnetic field, surrounds a small region of opposite magnetic polarity. This configuration leads to a situation where the source of the slow wind comes from the top edge of the coronal hole extension, then faster solar wind velocity arises as the magnetic field foot points move deeper into the coronal hole extension. The open field line foot points move closer to the opposite polarity region within the
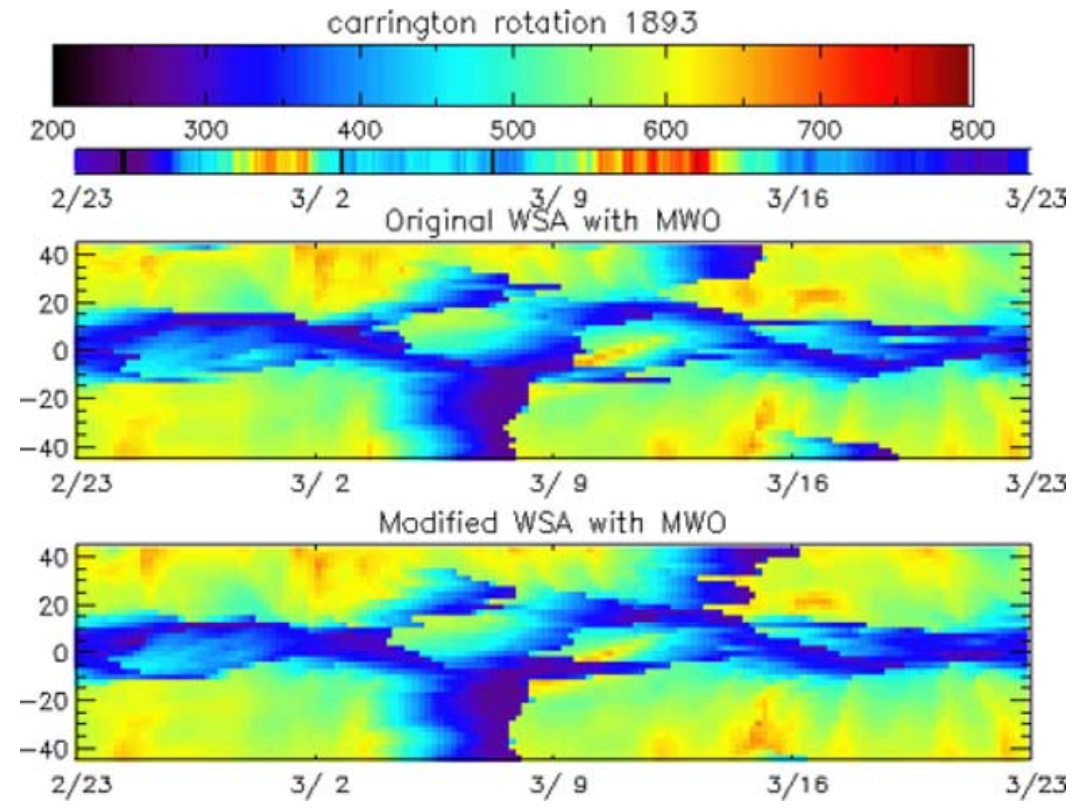

Figure 6. Solar wind velocity predictions at $1 \mathrm{AU}, 45^{\circ}$ above and below the ecliptic plane $\left(0^{\circ}\right)$ for $\mathrm{CR}$ 1893. The top color bar shows the velocity range. The second bar shows the wind observations for this time period. 
coronal hole extension, the predicted solar wind slows once more, followed by fast wind again as the source region moves deeper into the polar coronal hole. This transition between solar wind speeds as the field lines map along the coronal hole extension and into the polar hole, can be seen in the predicted solar wind speeds in Figure 6 from March 10 to 13 . Starting at an ecliptic latitude of $20^{\circ} \mathrm{N}$, and moving southward, we see the same pattern of slow wind, fast wind, slow wind, and fast wind which magnetically maps back to the coronal hole extension in the southern hemisphere.

[23] In the case of the first high-speed stream in the beginning of the Carrington rotation 1893 (February 28 to March 4) that both models failed to predict (Figure 3d), there is no indication in the latitude extended predictions (Figure 6) to indicate a high-speed stream at that time close to the ecliptic. A high-speed stream can be found in the Northern hemisphere beyond $20^{\circ}$ above the ecliptic, but this high-speed stream would have a positive magnetic polarity. The Wind observations indicate the solar wind had a negative magnetic polarity during this time, which both models do accurately reproduce. Therefore the lack of a high-speed wind predicted at earth for this time period cannot be explained by a latitudinal shift of the solar wind stream. The models simply do not predict its occurrence at any latitude during this rotation. This high-speed stream reoccurs in several rotations prior to Carrington rotation 1893 , for which the models accurately predict its presence, although underestimate it's speed and duration. Both models find that the source region for this wind maps back to the southern polar coronal hole around $330^{\circ}$ longitude in Figure 4. This missing stream is therefore not due to mapping of the earth-connected field line to an incorrect source region because of kinks at the interface surface. Comparisons with He I images do not match the southern polar coronal hole well at this longitude, indicating that the open field calculated in this region is in error. A better fit to the polar magnetic fields may produce a more accurate representation of the polar coronal holes (Arge et al. SPD conference, 2006 and publication in preparation) and thus a closer match between solar wind speed predictions and observations at 1 AU.

[24] Figure 6 provides a context for high-speed streams observed in the ecliptic. To validate these predictions, solar wind observations from other solar latitudes are required. Although we are unable to show whether the changes seen in solar wind velocity predictions (away from the ecliptic) are improvements or not, it is important to note that there are a few differences in the predicted velocity structure which also occur at higher latitudes. Those differences that do occur center around regions of slow solar wind located at high latitudes which map to 'sparse' regions within the polar coronal holes (e.g., $-90^{\circ}$ latitude, $270^{\circ}$ longitude or $90^{\circ}$ latitude $210^{\circ}$ longitude). Preliminary solar wind speed comparisons with Ulysses do not match these high latitude slow solar wind features, confirming that they are unphysical. These slow solar wind features at higher latitudes are believed to be due to artifacts in the routines contained within WSA, which calculate the open regions on the photosphere. Currently under development are improvements to these routines which may fill in these areas on the photosphere, increasing the predicted solar wind speed.
These improvements may very well remove these differences at higher latitudes associated with using the original and modified versions of the WSA model, thus confirming that changes to solar wind speed predictions and connectivity are most prevalent from sources close to the current sheet.

[25] In a few cases the modified WSA model solar wind velocity predictions are worse than those of the original WSA model, but generally not by much. An example of this is seen in Carrington rotation 1896 (Figure 3c) during the deceleration after the first high-speed stream. Figure 3e shows another example for Carrington rotation 1892. There are two time periods during this Carrington rotation with solar wind velocities above $600 \mathrm{~km} / \mathrm{s}$, from January 30 until February 5 and another where the solar wind velocity begins to increase on February 11, peaking around the February 13. Both models do poorly during the first highspeed stream, predicting solar wind velocities that begin around $500 \mathrm{~km} / \mathrm{s}$ but quickly decrease to around $400 \mathrm{~km} / \mathrm{s}$. Around February 10 both the original and the modified WSA model predict a second high-speed stream. The transition from slow wind to fast wind is too abrupt in both models, with the onset occurring a day early and peaking in 2 days, while it takes several days for the high-speed stream to peak in the observations. Both models predict velocities which are lower than observed for the second high-speed stream, and decelerate much sooner than is seen by Wind. On February 18 both models predict an increase in solar wind velocity to about $400 \mathrm{~km} / \mathrm{s}$, thus matching the solar wind observations. From February 18 to 21 the original WSA model more accurately predicts the solar wind velocity, where the modified WSA model consistently predicts about velocities $50 \mathrm{~km} / \mathrm{s}$ too slow.

[26] Overall the solar wind speed predictions of the modified WSA model are very similar to those of the original version over the whole of 1995, though there are occasions, as illustrated in Figure 3, where the new coupling improves the predictions significantly. In those cases where the old coupling approach does better, the differences between the two methods are generally minor. While the modified coupling does change the global coronal magnetic field configuration, it usually produces only small changes in the predicted solar wind speed. This can be understood by examining the equation used to calculate the solar wind velocity. This equation uses the expansion factor and depth of the open field line foot point in the coronal hole to derive the solar wind velocity. The WSA model can connect an open field to the photosphere in one of four ways: (1) connected deep within a coronal hole and with a large expansion factor, (2) connected deep within a coronal hole and with a small expansion factor, (3) connected near the edge of a coronal hole and with a large expansion factor, and (4) connected near the edge of a coronal hole and with a small expansion factor. The fourth of these possibilities produces solar wind velocity predictions that are the most sensitive to small changes in the expansion factor and coronal hole depth, especially if the foot point is within $2-4^{\circ}$ from the edge of the coronal hole. Large changes in predicted velocity can occur with the other three possibilities, but they require much larger differences in the expansion factor and/or coronal hole depth. Since the modification to the WSA model usually 
produces small changes to the magnetic configuration, and therefore to the expansion factors and coronal hole depth, significant changes in predicted velocity will usually occur in those situations with small expansion factors and small to intermediate coronal hole depths. Our analysis of the Carrington rotations shown in Figure 3 confirms that this is the case for rotations 1901 and 1902. In the other cases as shown in Figures $3 \mathrm{c}-3 \mathrm{e}$, differences in velocity are caused by unusually large changes in either coronal hole depth of the open field foot point or expansion factor.

\section{Skill Score Comparisons}

[27] Skill scores provide a means of quantifying the relative performance of models in reproducing observed values. Skill scores are computed by comparing the mean square error of both the original and modified model's predictions with that of a reference "baseline" model [e.g., Owens et al., 2005]. The baseline model for this purpose was the WSA model with $5^{\circ}$ National Solar Observatory at Kitt Peak (NSO) synoptic maps and no polar corrections.

[28] The computation of a skill scores for Carrington rotations 1890-1904 shows that the modification to WSA improves the model skill at predicting solar wind speed and IMF polarity at earth by $1.7 \%$ and $1.4 \%$ respectively. This increase in skill score shows that statistically the solar wind predictions for the original and modified models are very similar. The difference comes from occasional, significant improvements, and in general the predictions have not been worsened by this simple fix.

\section{Summary}

[29] In this paper we have modified the WSA model by introducing a second heliocentric surface across which the magnetic fields of the two component models are matched. This simple modification substantially reduces the kinks in the field lines observed in the original model at the point where the two solutions are matched, and hence substantially reduces the currents flowing on this boundary surface. This modification also changes the model's predictions of solar wind velocity at earth.

[30] We compared the solar wind velocity predictions of the original and modified WSA models for Carrington rotations 1890-1904, spanning the year 1995, to study the effects of minimizing the kinked field lines. This is an ideal period for this particular study, as the kinks found in the original model are most pronounced near the current sheet. Since, the current sheet was relatively flat during the year 1995, earth spent more time skimming along the current sheet than compared to other times of the solar cycle, thus making it more likely that the effects of minimizing the kinks would be captured in the comparison.

[31] Individual Carrington rotations were examined in detail and periods identified where the modification both improves and degrades the prediction, showing that small changes to the magnetic configuration can introduce large variations in predicted velocity. In general, we find that the solar wind speed predictions of the modified WSA model are very similar to those of the original version for the whole of 1995 . However, there are a number of important exceptions (e.g., CR 1901), lasting typically for only a few days, where the new coupling improves the model predictions significantly. In those cases where the old coupling approach does better than the new one, we find that the differences in the two methods are generally very minor. Therefore the simple fix yields solar wind speed predictions very similar to the old method most of the time, but occasionally, it produces a significant Improvement. This is born out statistically, where we find only a slight improvement of $1.7 \%$ in the overall skill score of solar wind speed for 1995 compared with the original model. One should only expect minor improvement in skill when the results do not change significantly except for occasional spikes in improvement. We find that the kinks in the original model do not generally significantly affect the predictions at $1 \mathrm{AU}$ even near solar minimum when one would expect to see the largest effects. This can be attributed to the form of the velocity equation. Only during certain situations is the velocity equation sensitive to small changes in the magnetic foot point position and flux tube expansion. Although, the solar wind velocity predictions were not affected (save for specific time periods) by the removal of the kinks from the PFSS and SCS interface, the differences may prove important for solar wind source region studies. The removal of the kinks can lead to changes in the connectivity, which predicts different source regions on the photosphere, which may or may not lead to changes in the predicted solar wind speed.

[32] On some occasions the model performs poorly such as the missing high-speed streams in CR 1892 and 1893. Since both the original and modified models perform essentially equally badly, we conclude that the discrepancies are not due to kinked field lines. We suggest three possible explanations for the discrepancies between the model predictions and the observations. First, it is well known that the coronal models are very sensitive to the strengths of polar fields [e.g., Hoeksema, 1984; Arge and Pizzo, 2000; Zhao et al., 2006; Arge and Owens, 2006; Liu et al., 2007], especially during solar minimum when they are strong. Recent work by one of us (Arge et al. SPD conference, 2006 and publication in preparation) reiterates this point, and shows how well the coronal holes obtained from models match observations depends on the polar field corrections. It may be that polar corrections applied to the photospheric field synoptic maps for Carrington maps from $1892-1893$ could be improved (the solar b angle is large and negative at this time and thus the north pole is poorly observed). This is a problem all coronal models face independent of their sophistication. The second possibility is that there may be a better velocity equation to use for any one rotation which would give the best fit to observations. Although the equation was calibrated to best fit the entire year of 1995, a different velocity equation, calibrated for a single rotation, may reveal these missed high-speed streams or alleviate overpredictions. However, the recalibration of each Carrington rotation individually introduces too many free parameters. The third possibility may be the assumption that the coronal magnetic fields are close to potential, which often breaks down near active regions [Schrijver et al., 2005]. This would lead to model magnetic fields which are locally not representative of the coronal fields. This would lead to erroneous velocity predictions at 1 AU. 
[33] Acknowledgments. We would like to thank R. Ulrich and the staff at Mount Wilson solar observatory for providing us access to their data. We have also benefited from the availability of the WIND and ACE data at NSSDC. This research was supported in part by the CISM project, which is funded by the STC Program of the National Science Foundation under the agreement ATM-0120950. This research was supported in part by the National Science Foundation under grant DGE-0221680.

[34] Zuyin Pu thanks Yi-Ming Wang and another reviewer for their assistance in evaluating this paper.

\section{References}

Altschuler, M. A., and G. Newkirk Jr. (1969), Magnetic fields and the structure of the solar corona, Sol. Phys., 9, 131-149.

Arge, C. N., and V. J. Pizzo (2000), Improvement in the prediction of solar wind conditions using near-real time solar magnetic field updates, J. Geophys. Res., 105(A5), 10,465-10,479.

Arge, C. N., and M. J. Owens (2006), Using solar and in situ observations to improve and constrain corona \& solar wind models, paper presented at AGU Fall Meeting, San Francisco, Calif.

Arge, C. N., D. Odstrcil, V. J. Pizzo, and L. R. Mayer (2003), Improved method for specifying solar wind speed near the sun, in Solar Wind Ten, vol. 679, edited by M. Velli, R. Bruno, and F. Malara, AIP Conf. Proc., p. 190, Melville, New York.

Arge, C. N., L. G. Luhmann, D. Odstrcil, C. J. Schrijver, and Y. Li (2004), Stream structure and coronal sources of the solar wind during May 12th, 1997 CME, J. Atmos. Sol. Terr. Phys., 66, 1295-1309.

de Toma, G., and C. N. Arge (2006), Multi-wavelength observations of coronal holes, in Proc. of the NSO Workshop 22, Large Scale Structures and Their Role in Solar Activity, edited by K. Sankarasubramanian, M. Penn, and A. Pevtsov, p. 251.

de Toma, G., C. N. Arge, and P. Riley (2005), Observed and modeled coronal holes, in Proc. of the Solar Wind 11/SOHO 16, Connecting Sun and Heliosphere, Whistler, Canada, p. 609, ESA Publications, Noordwijk, Netherlands.

Hoeksema, J. T. (1984), Structure and evolution of the large scale solar and heliospheric magnetic fields, Ph.D. thesis, 222 pp., Stanford Univ., Stanford, Calif.

Liu, Y., J. T. Hoeksema, X. Zhao, and R. M. Larson (2007), MDI synoptic charts of magnetic field: Interpolation of polar fields, AAS/AAPT Joint Meeting, Honolulu, Hawaii.
Neugebauer, M., et al. (1998), Spatial structure of the solar wind and comparisons with solar data and models, J. Geophys. Res., 103(A7), $14,587-14,599$

Neugebauer, M., P. C. Liewer, E. J. Smith, R. M. Skoug, and T. H. Zurbuchen (2002), Sources of the solar wind at solar activity maximum, J. Geophys. Res., 107(A12), 1488, doi:10.1029/2001JA000306.

Owens, M. J., C. N. Arge, H. E. Spence, and A. Pembroke (2005), An event-based approach to validating solar wind speed predictions: Highspeed enhancements in the Wang-Sheeley-Arge model, J. Geophys. Res., 110, A12105, doi:10.1029/2005JA011343.

Schatten, K. H. (1971), Current sheet magnetic model for the solar corona, Cosm. Electrodyn., 2, 232-245.

Schatten, K. H., J. M. Wilcox, and N. F. Ness (1969), A model of interplanetary and coronal magnetic fields, Sol. Phys., 6, 442-455.

Schrijver, C. J., M. L. DeRosa, A. M. Title, and T. R. Metcalf (2005), The nonpotentiality of active-region coronae and the dynamics of the photospheric magnetic field, Astrophys. J., 628, 501-513.

Wang, Y.-M., and N. R. Sheeley Jr. (1988), The solar origin of long-term variations of the interplanetary magnetic field strength, J. Geophys. Res., 93(A10), 11,227-11,236

Wang, Y.-M., and N. R. Sheeley (1990), Solar wind speed and coronal fluxtube expansion, Astrophys. J., 355, 726-732.

Wang, Y.-M., and N. R. Sheeley (1992), On potential field models of the solar corona, Astrophys. J., 392, 310-319.

Wang, Y.-M., and N. R. Sheeley (1995), Solar implications of Ulysses interplanetary field measurements, Astrophys. J., 447, L143.

Zhao, X. P., and J. T. Hoeksema (1993), A coronal magnetic field model with horizontal volume and sheet currents, Sol. Phys., 151, 91-105.

Zhao, X. P., and J. T. Hoeksema (1995), Prediction of the interplanetary magnetic field strength, J. Geophys. Res., 100(A1), 19-33.

Zhao, X. P., J. T. Hoeksema, Y. Liu, and P. H. Scherrer (2006), Success rate of predicting the heliospheric magnetic field polarity with Michelson Doppler Imager (MDI) synoptic charts, J. Geophys. Res., 111, A10108, doi:10.1029/2005JA011576.

C. N. Arge, Air Force Research Laboratory/Space Vehicles Directorate, Kirtland Air Force Base, NM, USA.

W. J. Hughes, S. L. McGregor, and M. J. Owens, Center for Integrated Space Weather Modeling, Boston University, 725 Commonwealth Avenue, Boston, MA 02215, USA. (slmic@bu.edu) 